Clare Dixon · Marcelo Finger (Eds.)

\title{
Frontiers of \\ Combining Systems
}

11th International Symposium, FroCoS 2017 Brasília, Brazil, September 27-29, 2017 Proceedings 


\section{Contents}

\section{Invited Talks}

Foundational (Co)datatypes and (Co)recursion for Higher-Order Logic. . . . . .

Julian Biendarra, Jasmin Christian Blanchette, Aymeric Bouzy, Martin Desharnais, Mathias Fleury, Johannes Hölzl, Ondřej Kunčar, Andreas Lochbihler, Fabian Meier, Lorenz Panny, Andrei Popescu, Christian Sternagel, René Thiemann, and Dmitriy Traytel

Designing Theory Solvers with Extensions. ................

Andrew Reynolds, Cesare Tinelli, Dejan Jovanović, and Clark Barrett

\section{Description and Temporal Logics}

A New Description Logic with Set Constraints and Cardinality

Constraints on Role Successors. . . . . . . . . . . . . . . . .

Franz Baader

Metric Temporal Description Logics with Interval-Rigid Names . . . . . . . Franz Baader, Stefan Borgwardt, Patrick Koopmann, Ana Ozaki, and Veronika Thost

Using Ontologies to Query Probabilistic Numerical Data . . . . . . . . . . .

Franz Baader, Patrick Koopmann, and Anni-Yasmin Turhan

Pushing the Boundaries of Reasoning About Qualified

Cardinality Restrictions . . . . . . . . . . . . . . . . . . . . . . .

Jelena Vlasenko, Volker Haarslev, and Brigitte Jaumard

\section{Rewriting}

Parallel Closure Theorem for Left-Linear Nominal Rewriting Systems . . . . . . Kentaro Kikuchi, Takahito Aoto, and Yoshihito Toyama

Complexity Analysis for Term Rewriting by Integer Transition Systems .... Matthias Naaf, Florian Frohn, Marc Brockschmidt, Carsten Fuhs, and Jürgen Giesl 


\section{SAT, SMT and Automated Theorem Proving}

Solving SAT and MaxSAT with a Quantum Annealer: Foundations

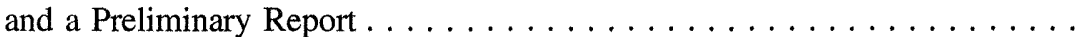

Zhengbing Bian, Fabian Chudak, William Macready, Aidan Roy,

Roberto Sebastiani, and Stefano Varotti

Superposition with Structural Induction $\ldots \ldots \ldots \ldots \ldots \ldots \ldots \ldots$

Simon Cruanes

Subtropical Satisfiability

Pascal Fontaine, Mizuhito Ogawa, Thomas Sturm, and Xuan Tung Vu

\section{Decision Procedures, Decidability and Verification}

On Solving Nominal Fixpoint Equations . . . . . . . . . . . . . . . 209

Mauricio Ayala-Rincón, Washington de Carvalho-Segundo,

Maribel Fernández, and Daniele Nantes-Sobrinho

Decidable Verification of Decision-Theoretic GoLog . . . . . . . . . . .

Jens Claßen and Benjamin Zarrieß

The Bernays-Schönfinkel-Ramsey Fragment with Bounded

Difference Constraints over the Reals Is Decidable Marco Voigt

\section{Properties and Combinations of Logic}

First-Order Interpolation of Non-classical Logics Derived from Propositional Interpolation

Matthias Baaz and Anela Lolic

Finitariness of Elementary Unification in Boolean Region

Connection Calculus ...........................

Philippe Balbiani and Çiğdem Gencer

Merging Fragments of Classical Logic. . . . . . . . . . . . . . . . . . 298

Carlos Caleiro, Sérgio Marcelino, and João Marcos

Interpolation, Amalgamation and Combination (The Non-disjoint

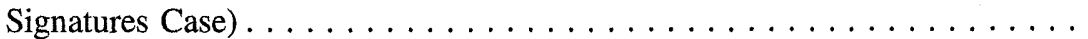

Silvio Ghilardi and Alessandro Gianola

The Boolean Solution Problem from the Perspective of Predicate Logic..... Christoph Wernhard 